\title{
Examples of Research Affecting the Practice of Networking
}

As networking researchers, we love to work on ideas that improve the practice of networking. In the early pioneering days of the Internet the link between networking researchers and practitioners was strong; the community was small and everyone knew each other. Not only were there many important ideas from the research community that affected the practice of networking, we were all very aware of them. Today, the networking industry is enormous and the practice of networking spans many network equipment vendors, operators, chip builders, the IETF, data centers, wireless and cellular, and so on. There continue to be many transfers of ideas, but there isn't a forum to learn about them. The goal of this series is to create such a forum by presenting articles that shine a spotlight on specific examples; not only on the technology and ideas, but also on the path the ideas took to affect the practice. Sometimes a research paper was picked up by chance; but more often, the researchers worked hand-in-hand with the standards community, the open-source community or industry to develop the idea further to make it suitable for adoption. Their story is here.

We are seeking CCR articles describing interesting cases of "research affecting the practice," including ideas transferred from research labs (academic or industrial) that became:

- Commercial products.

- Internet standards

- Algorithms and ideas embedded into new or existing products

- Widely used open-source software

- Ideas deployed first by startups, by existing companies or by distribution of free software

- Communities built around a toolbox, language, dataset

We also welcome stories of negative experiences, or ideas that seemed promising but ended-up not taking off.

In this issue, George Varghese describes his experience with technology transfer of network algorithms.

\author{
Bruce Davie, Christophe Diot, \\ Lars Eggert, Nick McKeown, \\ Venkat Padmanabhan, and Renata Teixeira \\ SIGCOMM Industrial Liaison Board
}

